\title{
Effectiveness of a Novel Calcium-enriched Mixture Root Cement to Decelerate Replacement Resorption in Replanted Teeth: A Case Report
}

\author{
Tony Francis ${ }^{1}$, Nasil Sakkir ${ }^{2}$, Vaibhav Kumar ${ }^{3}$, Sonal B Joshi ${ }^{4}$
}

\begin{abstract}
Aim: To assess the effectiveness of calcium-enriched mixture (CEM cement) to decelerate replacement resorption in replanted teeth. Background: A high prevalence of traumatic injuries in the orofacial region have been reported in school children. External replacement resorption (ERR) is the most common complication of replanting an avulsed tooth. Ankylosed teeth were lost in the first few years after ERR was initiated in young patients. Fixed replacement using dental implants or bridges is best delayed until skeletal growth is completed in young patients. With the gamut of biosilicate cements available, operators can now attempt to salvage these young permanent teeth diagnosed with ERR until the age when skeletal growth is complete.

Case description: In this case report, a novel biosilicate cement, CEM cement, was used to retard the progress of ERR and to preserve the affected teeth until the suitable age for receiving implants or bridges. In this 15-year-old patient, the resorptive process was regressed using CEM cement and the tooth remained functional till 22 years of age. The teeth were then extracted and implant treatment was initiated.

Conclusion: Currently there is no suitable protocol for the management of these cases. Newer biosilicate cements such as CEM cement help decelerate the resorptive progress and can be considered as a suitable protocol in intervening ERR.

Clinical significance: Losing an anterior tooth after ERR has functional, esthetic, phonetic, and psychological impacts on children and adolescents. Down regulating the resorptive process is critical and paramount in preserving esthetics and function until the time extraction and replacement can be done safely.

Keywords: Ankylosis, Avulsed tooth, Biosilicates, Calcium-enriched mixture cement, External replacement resorption.

World Journal of Dentistry (2019): 10.5005/jp-journals-10015-1679
\end{abstract}

\section{INTRODUCTION}

The reported prevalence of dentoalveolar trauma in children is as high as $34 \%{ }^{1}$ The young permanent anterior teeth are most commonly affected due to the susceptible position and labial proclination. ${ }^{2}$ This can lead to luxation injuries with pulpal and periapical sequelae. ${ }^{3}$ Repositioning the luxated teeth, nonrigid splinting, and endodontic therapy based on the pulpal status are included in the present guidelines for managing luxation injuries. ${ }^{4}$ In avulsion, the tooth is completely displaced out of its socket and the most common complication following replantation of avulsed teeth is external root resorption with a prevalence of $57-80 \% .^{5}$

External root resorption can be inflammatory resorption or replacement resorption. External inflammatory root resorption can be initiated by the infected necrotic pulp secondary to replantation of an avulsed tooth or a luxation injury. ${ }^{6}$ And ERR can initiate after replantation or luxation injuries, if injury to the periodontal ligament exceeds $20 \%$ of the periradicular area. ${ }^{3,7}$ When the periodontal ligament surrounding the root is damaged, the resulting proximity of the root to the consistently remodeling osseous tissues can lead to resorption by the clastic cells of the hematopoietic lineage. ${ }^{7}$

Conventional root canal therapy with an added regimen of calcium hydroxide $(\mathrm{CH})$ dressing has successfully arrested external inflammatory root resorption in $97 \%$ cases. ${ }^{5}$ On the contrary, there is no suitable protocol in the intervention of ERR. ${ }^{8,9}$ A Cochrane systematic review stated a lack of evidence towards a suitable protocol for managing external replacement root resorption. ${ }^{10}$

External replacement resorption in adults can progress extremely slow, allowing the teeth to function for decades
${ }^{1}$ Department of Conservative Dentistry and Endodontics, Melaka Manipal Medical College, Manipal Academy of Higher Education, Melaka, Malaysia

${ }^{2}$ Department of Endodontics, Trien Dentistry-The Dental Clinic, Kochi, Kerala, India

${ }^{3}$ Department of Public Health Dentistry, Terna Dental College, Navi Mumbai, Maharashtra, India

${ }^{4}$ Department of Conservative Dentistry and Endodontics, KLE Vishwanath Katti Institute of Dental Sciences, Belagavi, Karnataka, India

Corresponding Author: Tony Francis, Department of Conservative Dentistry and Endodontics, Melaka Manipal Medical College, Manipal Academy of Higher Education, Melaka, Malaysia, Phone: +60176545281, e-mail: tonyfrancier@gmail.com

How to cite this article: Francis T, Sakkir N, Kumar V, et al. Effectiveness of a Novel Calcium-enriched Mixture Root Cement to Decelerate Replacement Resorption in Replanted Teeth: A Case Report. World J Dent 2019;10(6):457-460.

Source of support: Nil

Conflict of interest: None

or a lifetime. ${ }^{5}$ On the contrary, ERR, during the period of active skeletal growth, leads to loss of teeth within 1-5 years' period. ${ }^{5}$ Loss of young permanent teeth imposes difficulty in replacing them until skeletal growth is completed..$^{11}$ Implants and bridges can disturb the normal growth pattern of alveolar bone and not 
synchronize with the eruption of adjacent teeth. Waiting with interim dentures till the growth phase is complete can lead to postextraction resorption of the alveolar bone. ${ }^{12}$ Current guidelines aim at the prevention of ERR by reducing extraoral dry time and placing the most desirable flexible splinting for replanted teeth for short durations (up to 2 weeks). Extraoral dry time and the extent of injury to the periodontium are often beyond control.

Literature search on domain-specific portals such as PubMed and EBSCO, using the targeted and focused PICO stem through the key words, yielded no definite scientific sound guideline that has been proposed in the management of a replanted tooth once replacement resorption is initiated..$^{13}$ Although submergence and infraocclusion of the ankylosed teeth occurred when the teeth were traumatized before 16 years of age in boys and 14 years in girls, it is critical and of paramount importance to conservatively manage and preserve these teeth until the time it is safe to consider implants or bridges for the young patients. ${ }^{11}$ Efforts should be taken through evidence-informed healthcare practices to dampen and decelerate the consequences and squeal of reimplantation. We present a detailed case of management of external replacement root resorption in replanted teeth that was systematically decelerated using a novel biosilicate, CEM.

\section{Case Description}

This case report has been described using the CARE (CAse REport) guidelines, in tandem with the equator network, for enhancing the quality and transparency of health research. A 15-year-old male patient presented to the specialist clinic (Department of Conservative Dentistry and Endodontics, KLE VK Institute of Dental Sciences, Belgaum, Karnataka, India) with complaints of pain and discomfort in the upper front teeth. Patient had a history of dentofacial trauma during sports, which resulted in avulsion of teeth 11, 12, and 21 (Federation Dentaire Internationale (FDI) tooth notation system). He had visited a nearby general practice within 2 hours and the teeth were replanted and stabilized by a routinely done splinting procedure. Root canal treatment was performed within 2 weeks. With the persistent symptoms even after 4 months following replantation of avulsed front teeth, the parents brought the young patient to the specialist clinic.

Patient complained of a gnawing, dull, and lingering pain in that region ever since teeth reimplantation. Clinical and radiographic examinations were carried out. On intraoral examination, a steel wire and composite resin splint were noticed in place. Increased probing depths and gingival bleeding were noted for the affected teeth and the adjacent soft tissues were tender on palpation. No tenderness on vertical percussion was observed. Intraoral periapical radiograph showed that the replanted teeth had poor quality root canal fillings. Multiple resorptive lesions were evident around the roots of teeth 11, 12, and 21 (Fig. 1). Tooth 22 presented with an uncomplicated fracture of the crown with no evident resorptive lesions of the root but did not respond to electric and cold tests. Horizontal bone loss was seen on the periapical radiograph, bordering the affected region. The adjacent teeth responded normally to electric and cold tests.

Periodontal ligament space could not be traced around most areas of the roots of the replanted teeth. Direct union between the roots and the alveolar bone was suggestive of replacement resorption. A distinct high percussive tone was noted for the affected teeth. Physiologic mobility was lost, when compared to adjacent healthy teeth. With the absence of infected pulp and associated inflammatory lesions in the periradicular bone, a diagnosis of external inflammatory root resorption was ruled out

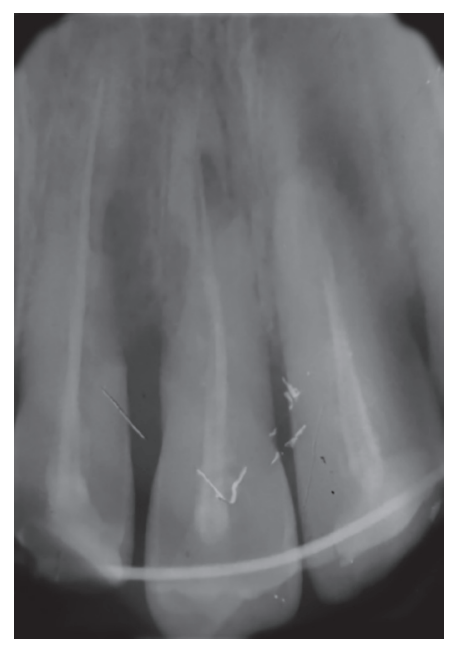

Fig. 1: Pretreatment radiograph

and a diagnosis of external replacement root resorption (ERR)/ ankylosis was drawn. Patient was informed regarding the inevitable tooth loss in the next few years. The young patient and the parents were equally perplexed and sought immediate intervention. Shortfalls of performing osseointegrated implants or crown and bridge prosthesis after immediate extraction during the growing age were explained. After being guided through a well-informed written consent, the treatment was initiated to retain the resorbing teeth as long as possible before extraction and replacement would be deemed necessary.

After rubber dam isolation using split dam technique, access cavities were prepared for the affected teeth. The existing root canal fillings were removed using NiTi retreatment files (ProTaper Retreatment files, Dentsply Maillefer, OK, USA) and a guttapercha solvent (Endosolv ${ }^{\mathrm{TM}}$; Septodont, France). Working length was reestablished using radiographs (Fig. 2) and the canals were prepared using ProTaper Next files ${ }^{\mathrm{TM}}$ (Dentsply Maillefer, OK, USA). Sodium hypochlorite of $0.5 \%$ and EDTA solution were intermittently used for thorough irrigation. After drying the canals, CEM cement (Bionique Dent, Tehran, Iran) was used to fill the canal entirely, using prefitted pluggers (Dentsply Maillefer, OK, USA). A moist cotton pellet was placed over the canal orifice and the access cavity was temporarily restored with glass ionomer cement (Fuji II LC; GC Corp., Tokyo). The pre-existing splint was carefully removed, and the teeth were cleaned and polished. After 2 days, the cotton pellet was removed and the access cavity was restored with a bonded composite resin restoration (Filtek Z350 XT, 3M ESPE, St. Paul, MN, USA) (Fig. 3). The nonvital tooth 22 with the fractured crown was treated by performing a conventional root canal treatment followed by glass fiber post placement and a composite resin restoration.

Follow-up appointment was scheduled after 3 weeks, per the protocol and it was found that the replanted teeth were asymptomatic. The gingival inflammation had subsided. Periapical radiograph showed that the resorptive lesions did not progress further. Follow-up appointments were scheduled every 6 months for up to 2 years. The patient did not make follow-up visits as scheduled and was seen after a period of 18 months. The resorptive lesions were evident on the central incisors but seemed to have progressed slowly after treatment (Fig. 4) and the teeth were asymptomatic. Since the patient was relocating for further studies, he was asked to inform us if there was pain, mobility, or any discomfort in the region. Telephonic review was sought 


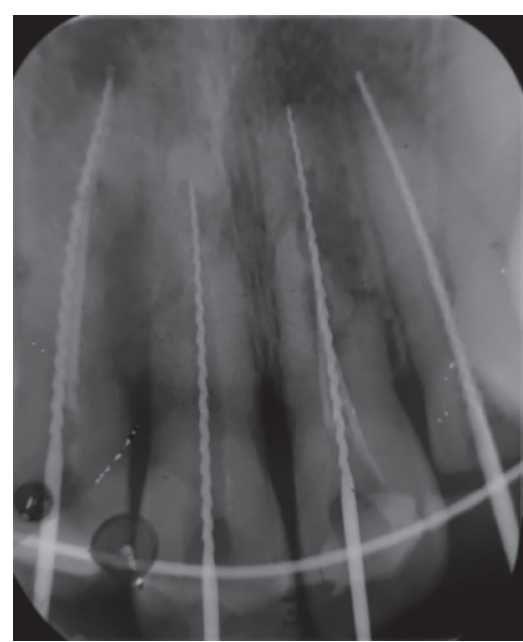

Fig. 2: Retreatment radiograph showing reestablishment of working length in relation to $11,12,21$, and 22

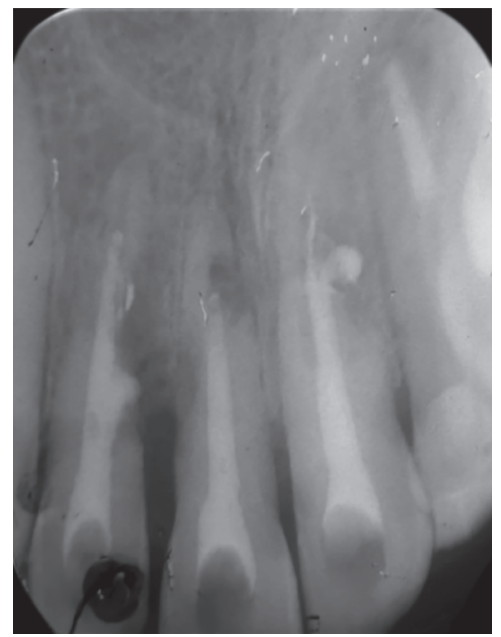

Fig. 4: Follow-up radiograph 18 months after treatment

every 6 months and the teeth reportedly remained asymptomatic and functional until 22 years of age; after which he reported that the teeth had become mobile. On visiting a nearby dentist, the mobile teeth were extracted and treatment with osseointegrated implants were initiated safely as skeletal growth was deemed to be completed at that age.

\section{Discussion}

Most luxation injuries require immediate repositioning and shortterm splinting with a nonrigid material. A systematic review and meta-analysis stated that composite resin and wire splinting resulted in a threefold increase in replacement resorption when compared to a more flexible suture splinting. ${ }^{14}$ Multiple studies have reported adverse outcome of long duration of splinting of repositioned/replanted teeth. ${ }^{15}$ The International Association of Dental Traumatology guidelines endorse a nonrigid functional splinting of repositioned teeth for 2-4 weeks' time, in view of this conclusive evidence. In this case, the long duration of placing a relatively rigid splint could have accelerated the resorptive process.

Management of ERR was often based on operator experiences and patient-related factors. ${ }^{16}$ The widespread clinical success of

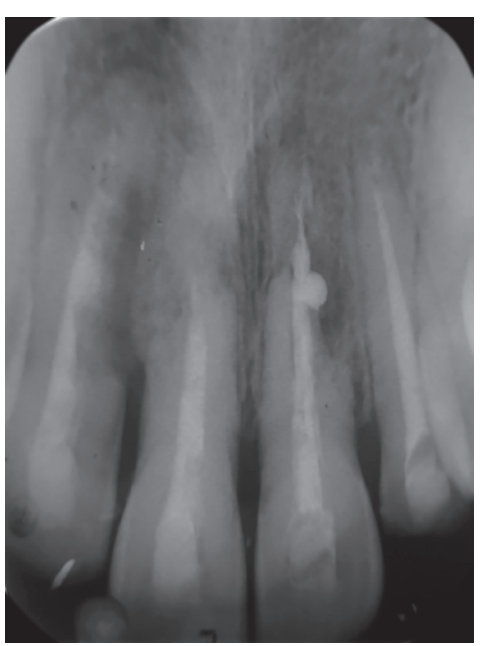

Fig. 3: Radiograph after placement of calcium-enriched mixture (CEM) cement in root canals of teeth 11,12 , and 21

implants in replacing missing teeth in adults often persuades the clinician to plan extraction of resorbing teeth and implant placement in young patients. Implant placement in growing children can disturb normal bone growth. ${ }^{17}$ Implant position in relation to other teeth can change with growth-related movements of jaw bones often leading to relative infraocclusion or labioversion. Although chronological age may not be a suitable standard for evaluating skeletal growth cessation, it has been estimated that craniofacial growth can proceed till the age of 15 years in girls and up to 17 years of age in boys. ${ }^{17}$ Estimating skeletal age using hand wrist radiographic technique or cephalometric analysis at regular intervals are more precise in identifying skeletal growth cessation. ${ }^{17}$ There is consensus in delaying implant placement until craniofacial/ skeletal growth is completed.

Although ankylosed teeth do not harmonize with the normal growth of the alveolar bone or with the eruption of adjacent teeth, they help to maintain the functional and Esthetic demands of the patient along with preventing postextraction resorption of the alveolar bone. Early extraction and orthodontic closure of space, intentional replantation after soaking the roots in fluoride solutions and Emdogain ${ }^{\mathrm{TM}}$ (Biora), autotransplantation of premolars, and decoronation followed by esthetic space maintainers are the reported treatment protocols attempted in managing ERR in children and adolescents. ${ }^{13}$ Along with the lack of evidence of success from long-term follow-up, these procedures carry the risk of further damage to the alveolar bone. ${ }^{13}$

During dentofacial trauma, damage to the nonmineralized structures over the root exposes the root dentin to the clastic cells. ${ }^{18}$ During active inflammation, cytokines, especially interleukin-1 $\beta$, have been shown to induce clastic cell genesis from precursor cells. ${ }^{9}$ An alkaline and osteoinductive biomaterial in close proximity can abate this upregulation of clastic cells. ${ }^{19}$ Calcium hydroxide was traditionally placed as intracanal dressings to deter replacement resorption. The mechanism of action of $\mathrm{CH}$ includes the release of $\mathrm{Ca}^{+}$and $\mathrm{OH}^{-}$ions which can permeate the dentinal tubules to create an alkaline environment. ${ }^{20}$ But long-term use of $\mathrm{CH}$ in the root canals can increase the brittleness of the root dentin, thereby increasing the risk of cervical root fractures, especially in immature teeth. ${ }^{21}$ Newer biosilicate cements surpass most known drawbacks of long-term dressing with $\mathrm{CH}$. Mineral trioxide aggregate (MTA) was the first biosilicate cement that was developed and widely used. 
Release of $\mathrm{Ca}^{+}$and $\mathrm{OH}^{-}$ions from biosilicate cements helps maintain an alkaline $\mathrm{pH}$, making it nonconducive for resorption to progress. ${ }^{22}$ Along with maintaining an alkaline $\mathrm{pH}$ for extended periods of time, these cements have the potential to stimulate cementoblasts and odontoblasts. ${ }^{23}$ They have excellent sealing property and biocompatibility. ${ }^{24}$ Panzarini et al. ${ }^{25}$ demonstrated smaller areas of ankylosis when MTA was used as root filling material in comparison with $\mathrm{CH}$, in a study involving delayed replantation of teeth in monkeys.

The newer CEM cement surpasses most known drawbacks of MTA such as tooth discoloration, poor handling characteristics, and slow setting time. A large body of evidence shows the desirable properties of CEM cement among the new biosilicate cements. ${ }^{26-28}$ Asgary et al. ${ }^{29}$ reported successful management of inflammatory external root resorption (IERR) in an avulsed tooth of a young patient using CEM cement. Healing of a progressive IERR with reestablishment of normal periodontal condition occurred within 40 months. $^{29}$ In the present case, the teeth diagnosed with ERR remained functional for 7 years before extraction and replacement with osseointegrated implants.

\section{Conclusion}

Currently there is no established guidelines to intervene external replacement root resorption. Loss of teeth can happen in the first few years after ERR is initiated in children and adolescents. Prosthetic replacement of these teeth including bridges or osseointegrated implants cannot be performed until skeletal growth is completed. The CEM cement has good biocompatibility, cost-effectiveness, and better physical properties compared to other biosilicate cements. The CEM cement can be used to decelerate the resorptive progress and intervene external replacement root resorption.

\section{References}

1. Hamilton FA, Hill FJ, Holloway PJ. An investigation of dento-alveolar trauma and its treatment in an adolescent population. Part 1: the prevalence and incidence of injuries and the extent and adequacy of treatment received. Br Dent J 1997;182(3):91-95. DOI: 10.1038/ sj.bdj.4809313.

2. Gassner R, Bosch R, Tuli T, et al. Prevalence of dental trauma in 6000 patients with facial injuries: implications for prevention. Oral Surg Oral Med Oral Pathol Oral Radiol Endod 1999;87(1):27-33. DOI: 10.1016/S1079-2104(99)70290-8.

3. Pohl Y, Wahl G, Filippi A, et al. Results after replantation of avulsed permanent teeth. III. Tooth loss and survival analysis. Dent Traumatol 2005;21(2):102-110. DOI: 10.1111/j.1600-9657.2004.00299.x.

4. Diangelis AJ, Andreasen JO, Ebeleseder KA, et al. International Association of Dental Traumatology guidelines for the management of traumatic dental injuries: 1 . Fractures and luxations of permanent teeth. Dent Traumatol 2012;28(2):2-12. DOI: 10.1111/j.16009657.2011.01103.x.

5. Finucane D, Kinirons MJ. External inflammatory and replacement resorption of luxated, and avulsed replanted permanent incisors: a review and case presentation. Dent Traumatol 2003;19(3):170-174. DOI: 10.1034/j.1600-9657.2003.00154.x.

6. Fuss Z, Tsesis I, Lin S. Root resorption--diagnosis, classification and treatment choices based on stimulation factors. Dent Traumatol 2003;19(4):175-182. DOI: 10.1034/j.1600-9657.2003.00192.x.

7. Pohl Y, Filippi A, Kirschner H. Results after replantation of avulsed permanent teeth. II. Periodontal healing and the role of physiologic storage and antiresorptive-regenerative therapy. Dent Traumatol 2005;21(2):93-101. DOI: 10.1111/j.1600-9657.2004.00298.x.

8. Malmgren B. Decoronation: how, why, and when? J Calif Dent Assoc 2000;28(11):846-854.
9. Gunraj MN. Dental root resorption. Oral Surg Oral Med Oral Pathol Oral Radiol Endod 1999;88(6):647-653. DOI: 10.1016/S10792104(99)70002-8.

10. de Souza RF, Travess $H$, Newton $T$, et al. Interventions for treating traumatised ankylosed permanent front teeth. Cochrane Database Syst Rev 2010;1:CD007820. DOI: 10.1002/14651858.CD007820.pub2.

11. Kawanami M, Andreasen JO, Borum MK, et al. Infraposition of ankylosed permanent maxillary incisors after replantation related to age and sex. Endod Dent Traumatol 1999;15(2):50-56. DOI: 10.1111/j.1600-9657.1999.tb00752.x.

12. Agarwal N, Kumar D, Anand A, et al. Dental implants in children: a multidisciplinary perspective for long-term success. Natl J Maxillofac Surg 2016;7(2):122-126. DOI: 10.4103/0975-5950.201362.

13. Sapir S, Shapira J. Decoronation for the management of an ankylosed young permanent tooth. Dent Traumatol 2008;24(1):131-135. DOI: 10.1111/j.1600-9657.2006.00506.x.

14. Chung W-C, Tu Y-K, Lin Y-H, et al. Outcomes of autotransplanted teeth with complete root formation: a systematic review and meta-analysis. J Clin Periodontol 2014;41(4):412-423. DOI: 10.1111/jcpe.12228.

15. Kahler B, Hu J-Y, Marriot-Smith CS, et al. Splinting of teeth following trauma: a review and a new splinting recommendation. Aust Dent J 2016;61(Suppl 1):59-73. DOI: 10.1111/adj.12398.

16. Ahangari $Z$, Nasser $M$, Mahdian $M$, et al. Interventions for the management of external root resorption. Cochrane Database Syst Rev 2015;11:CD008003. DOI: 10.1002/14651858.CD008003.pub3.

17. Op Heij DG, Opdebeeck $H$, van Steenberghe $D$, et al. Age as compromising factor for implant insertion. Periodontol 2000 2003;33:172-184. DOI: 10.1046/j.0906-6713.2003.03314.x.

18. Mehra N, Yadav M, Kaushik M, et al. Clinical management of root resorption: a report of three cases. Cureus 2018;10(8):e3215. DOI: 10.7759/cureus.3215.

19. Saberi E, Farhad-Mollashahi N, Sargolzaei Aval F, et al. Proliferation, odontogenic/osteogenic differentiation, and cytokine production by human stem cells of the apical papilla induced by biomaterials: a comparative study. Clin Cosmet Investig Dent 2019;11:181-193. DOI: 10.2147/CCIDE.S211893.

20. Carrotte P. Endodontics: part 9. Calcium hydroxide, root resorption, endo-perio lesions. Br Dent J 2004;197(12):735-743. DOI: 10.1038/ sj.bdj.4811897.

21. Andreasen JO, Farik B, Munksgaard EC. Long term calcium hydroxide as a root canal dressing may increase risk of root fracture. Dent Traumatol 2002;18(3):134-137. DOI: 10.1034/j.1600-9657. 2002.00097.x.

22. Aggarwal V, Singla M. Management of inflammatory root resorption using MTA obturation - a four year follow up. Br Dent J 2010;208(7):287-289. DOI: 10.1038/sj.bdj.2010.293.

23. Fridland M, Rosado R. MTA solubility: a long term study. J Endod 2005;31(5):376-379. DOI: 10.1097/01.DON.0000140566.97319.3e.

24. Asgary S, Eghbal MJ, Parirokh M. Sealing ability of a novel endodontic cement as a root-end filling material. J Biomed Mater Res A 2008;87(3):706-709. DOI: 10.1002/jbm.a.31678.

25. Panzarini SR, Sonoda CK, Saito CTMH, et al. Delayed tooth replantation: MTA as root canal filling. Braz Oral Res 2014;28:1-7. DOI: 10.1590/1807-3107BOR-2014.vol28.0059.

26. Utneja S, Nawal RR, Talwar S, et al. Current perspectives of bio-ceramic technology in endodontics: calcium enriched mixture cement review of its composition, properties and applications. Restor Dent Endod 2015;40(1):1-13. DOI: 10.5395/rde.2015.40.1.1.

27. Rahimi S, Mokhtari H, Shahi S, et al. Osseous reaction to implantation of two endodontic cements: mineral trioxide aggregate (MTA) and calcium enriched mixture (CEM). Med Oral Patol Oral Cir Bucal 2012;17(5):e907-e911. DOI: 10.4317/medoral.18136.

28. Asgary S, Ahmadyar M. One-visit endodontic retreatment of combined external/internal root resorption using a calcium-enriched mixture. Gen Dent 2012;60(4):e244-e248.

29. Asgary S, Nosrat A, Seifi A. Management of inflammatory external root resorption by using calcium-enriched mixture cement: a case report. J Endod 2011;37(3):411-413. DOI: 10.1016/j.joen.2010.11.015. 